\title{
Metastatic clostridial myonecrosis secondary to perforated metastatic bowel cancer
}

\author{
Nasser Mohammed Amer ${ }^{1}$, John Karayanis ${ }^{2}$ \\ ${ }^{1}$ King Fahad Hospital of the University, Al Khobar 31952, Saudi Arabia. \\ ${ }^{2}$ Locum Consultant General Surgery, Hereford County Hospital, Hereford HR1 2ER, United Kingdom. \\ Correspondence to: Dr. Nasser Mohammed Amer, King Fahad Hospital of the University, P O Box 40262, Al Khobar 31952, Saudi Arabia. \\ E-mail: nasser@nasseramer.com
}

How to cite this article: Amer NM, Karayanis J. Metastatic clostridial myonecrosis secondary to perforated metastatic bowel cancer. J Cancer Metastasis Treat 2016;2:402-6.

\author{
Article history: \\ Received: 05-04-2016 \\ Accepted: 06-07-2016 \\ Published: 21-10-2016 \\ Key words: \\ Metastatic gangrene, \\ perforated sigmoid cancer, \\ myonecrosis, \\ clostridia
}

\begin{abstract}
Spontaneous metastatic clostridial myonecrosis is a rare condition caused by Clostridium septicum. The underlying lesion is usually either a colonic neoplasm or leukemia. The authors reported a 67-year-old female who presented with acute abdomen secondary to a perforated sigmoid cancer and who developed gas gangrene in her right leg. Unfortunately, despite all resuscitative measures, she died. The authors reviewed the literature; the diagnosis of metastatic myonecrosis was based on a high index of suspicion, development of bullae containing gram-positive rods, and subcutaneous crepitus (although this was a late sign). Treatment involves aggressive fluid replacement, high doses of intravenous penicillin, high concentration of oxygen, and surgical debridement, and/or amputation. The mortality remains very high, despite all the above measures.
\end{abstract}

\section{INTRODUCTION}

Metastatic clostridial myonecrosis is an uncommon complication of malignancy, particularly of the gastrointestinal tract, and of leukemia. Without treatment the mortality rate reaches $100 \%$ within $48 \mathrm{~h}^{[1-4]} \mathrm{A}$ number of reports have demonstrated the association between atraumatic clostridial infection and internal malignancy. ${ }^{[5]}$

We reviewed the literature, which demonstrated the paramount importance of early diagnosis and institution of early aggressive management. We reported a case of a 67-year-old woman who developed sudden myonecrosis in her right thigh secondary to perforated large bowel cancer.

\section{CASE REPORT}

A 67-year-old female was admitted to a district general hospital via a general practitioner referral, complaining mainly of pain in the epigastrium for the past four days. Pain became worse and more constant on the day of

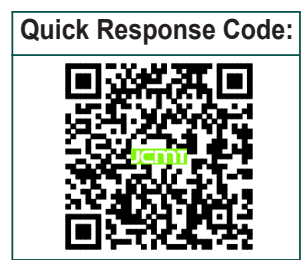


admission, radiating to her chest. The patient claimed milder episodes of similar attacks for the past four months. The pain was associated with nausea, but no vomiting, no change in her bowel habits, and no significant weight loss.

Her past medical history involved rheumatoid arthritis, hypertension, and rheumatic heart disease. She was receiving azathioprine and bendroflouazide for her hypertension.

On examination, the patient appeared toxic, pale but not jaundiced, and very restless. She had a temperature of $38.7^{\circ} \mathrm{C}$, her blood pressure was $108 / 61 \mathrm{mmHg}$, and her pulse was 100 per minute, regular but weak. Examination of her heart revealed a fine diastolic murmur and a small splinter hemorrhage in the right ring finger. There was no evidence of heart failure. Abdominal examination revealed tenderness in the epigastric area and guarding in the right upper quadrant along with a palpable left lobe of the liver. Rectal examination was normal with no evidence of blood.

Results of the patient's biochemical tests showed sodium 128, potassium 3 , urea 5 , creatinine 83 , aspartate transaminase 66 , alanine transaminase 25 , layered double hydroxide 781 , alkaline phosphatase $150, C$ reactive protein 351 , bilirubin 30 , hemoglobin 8.8 , and white blood count 5,300. The chest and abdominal radiographs were normal with no evidence of air under the diaphragm. The initial impression was of possible acute cholecystitis or peritonitis. The patient was resuscitated with intravenous fluids and oxygen and was given intravenous penicillin, gentamicin, and clindamycin.

Despite the aggressive resuscitation, the patient's condition deteriorated. A small area of dusky blue discoloration about $6 \mathrm{~cm} \times 4 \mathrm{~cm}$ appeared in the right popliteal fossa; this area was noticed to expand gradually. The leg became increasingly painful, and bullae appeared in the same area. Fine subcutaneous crepitation was noted in the same leg and was confirmed by plain X-ray [Figure 1], demonstrating gas in the soft tissue. Diagnosis of gas gangrene was established based on an aspirate from one of the blisters, which revealed gram-positive rods. Orthopedic involvement was sought; a decision was made to take the patient for hind-quadrant amputation, along with an exploratory laparotomy and possible Hartmann's procedure. Unfortunately, the patient did not survive the anesthesia and experienced cardiac arrest during induction.

The postmortem study revealed a perforated $4-\mathrm{cm}$ sigmoid colon cancer with evidence of peritonitis, and a second primary cecal cancer $6 \mathrm{~cm}$ in diameter. There was evidence of metastases in liver, lungs, and paraaortic nodes. The report also confirmed the presence of gas gangrene in the right thigh as well as the presence of subacute bacterial endocarditis.

\section{DISCUSSION}

Nontraumatic clostridial myonecrosis secondary to an underlying bowel cancer is a fulminant and often fatal infection caused by Clostridium septicum..$^{[4,6]}$ This is in contrast to most other cases of clostridial septicemia which are caused by Clostridium perfringens. ${ }^{[1,7]}$ The mortality is high even with aggressive management, and it can reach $100 \%$ if not treated within 48 h. ${ }^{[3,4]}$

In a review by Kornbuth et al..$^{[7]}$ of 162 cases of spontaneous $C$. septicum infection from the years 1945 to $1987,34 \%$ of patients had colorectal cancer while $40 \%$ had a hematologic malignancy. In $37 \%$ of the patients, the malignancy had not been diagnosed. Distant myonecrosis had an even greater association with occult colon cancer (see Table 1 for causes of metastatic clostridial gangrene ${ }^{[8]}$ ).

\section{Pathogenesis}

Myonecrosis is caused by Clostridia organisms, which are gram-positive rods that sporulate and are found in the soil..$^{[1,3,7]}$ C. septicum is more aerotolerant ${ }^{[8]}$ and the inoculum required for infection is 300 times smaller than that of C. perfringens. ${ }^{[7]}$ It is commonly found as a normal inhabitant of the gastrointestinal and genitourinary tracts. ${ }^{[1,9]}$ The spores usually exist for years and vegetate when conditions become optimal. C. septicum, however, is an opportunistic pathogen in humans, gaining entry to the bloodstream via breaches in the mucosa. This may be due to tumor necrosis ${ }^{[4]}$ or necrotizing colitis in patient with leukemia

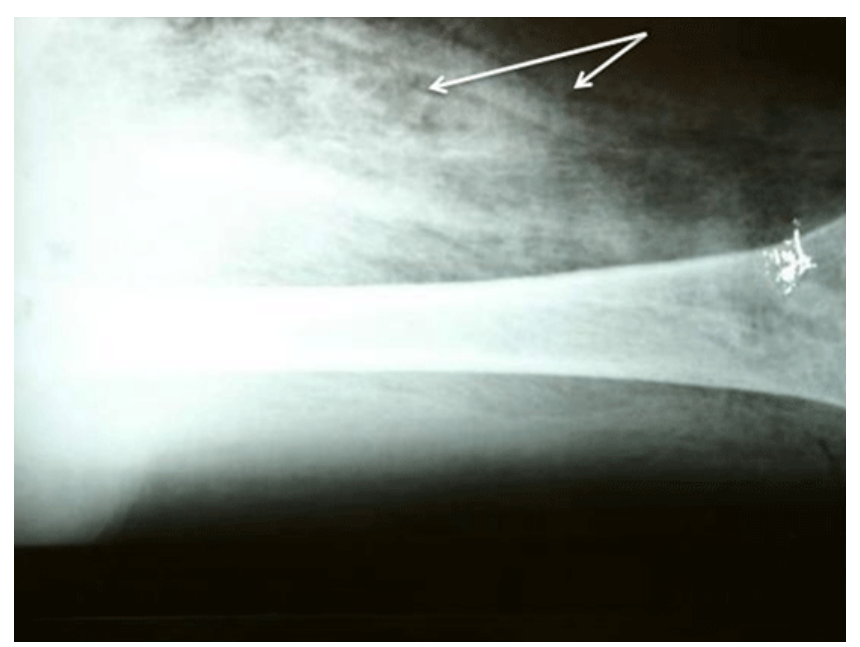

Figure 1: Plain radiograph of the right thigh. Arrows show subcutaneous gas from gas-forming organism 
Table 1: Underlying conditions in nontraumatic clostridial gangrene $^{[8]}$

\begin{tabular}{l}
\hline Gastrointestinal factors \\
\hline Colon tumor \\
Ulceration of mucosa \\
Chemotherapy \\
Radiation \\
Instrumentaion \\
Bowel infarction \\
Intestinal surgery \\
Diverticulitis \\
Necrotizing enterocolitis \\
Ileitis or colitis \\
Fecal impaction \\
Intussusception \\
Volvulus \\
Straining at stool \\
Systemic factors \\
Leukemia \\
Lymphoproliferative disorder \\
Diabetes mellitus \\
Metastatic tumor (nongastrointestinal) with chemotherapy \\
Neutropenia \\
Cirrhosis
\end{tabular}

or cyclic neutropenia. ${ }^{[5]}$ Presumably, the anaerobic glycolysis and the acidic milieu within a tumor provide a favorable environment for the germination of the clostridial spores. ${ }^{[5]}$ The affinity for necrotic tissue is not specific for malignant tissue only, as demonstrated by Thiele et al. ${ }^{[5]}$ where spores of Clostridia were injected in necrotic tissue other than tumor and were found to germinate. This study may explain the predilection of C. septicum for patients with necrotic colon cancer, spontaneous bowel perforation, chemotherapy treatment, surgery, or medical procedure such as endoscopy or barium enema. Once established, $C$. septicum may either cause a locally invasive infection or spread via the bloodstream to distant skeletal muscle (causing myonecrosis) or to other organs ${ }^{[4]}$ (producing abscesses that may be indistinguishable from metastasis). ${ }^{[3]}$ Clostridial organisms produce toxins that are responsible for the rapid spread and systematic toxicity of these infections. ${ }^{[3]} \mathrm{C}$. septicum is believed to produce four toxins, one of which is hemolysin, which is oxygen stable. In addition, it also produces a deoxyribonuclease, a hyalurinidase, and oxygen labile hemolysins. Secondary toxicity may result from the products of tissue breakdown such as creatinine phosphokinase (CPK). ${ }^{[3]}$ Diabetics, ${ }^{[5,6,9]}$ on the other hand, seem susceptible to developing spontaneous gas gangrene. This is most likely due to their propensity to develop focal tissue ischemia and acidosis secondary to atherosclerosis and microangiopathic vascular disease, which allow the circulating Clostridia organism to propagate in the hypoxic area. On the other hand, suppurative infection without signs of myonecrosis or toxemia is the most common form of clostridial disease..$^{[5]}$

The following is a histotoxic classification of gas gangrene (based on MacLennan's monograph): ${ }^{[5]}$
I. Traumatic:

A. Simple contamination (no clinical evidence of sepsis)

B. Anaerobic cellulitis (local gas gangrene, with healthy muscle not invaded, e.g. pressure sores, diabetic foot ulcer)

C. Anaerobic myonecrosis (classical, with invasion of living muscle)

II. Nontraumatic or idiopathic ${ }^{[9,11]}$ (typically arising from visceral intra-abdominal catastrophes, such as perforated cecal cancer)
A. Anaerobic cellulitis
B. Contiguous myonecrosis
C. Metastatic myonecrosis

\section{Clinical course}

Gas gangrene is a rapidly spreading infection. ${ }^{[7]}$ It can advance as fast as $2 \mathrm{~cm}$ per hour. The incubation period varies from $6 \mathrm{~h}$ to 2 days. ${ }^{[1]}$ The bacilli produce several exotoxins, which can destroy the host tissue and increase permeability. The resultant necrosis, edema, and ischemia favor clostridial reproduction in which more toxins are released, and a cycle ensues.

Carbon dioxide and hydrogen are liberated during the process, which opens fascial planes and facilitates spread. The pathogenesis of subcutaneous emphysema from disruption of the gastrointestinal tract depends on localized bowel wall weakness, ${ }^{[12]}$ the anatomic site, and an increased pressure gradient between the bowel lumen and extramural tissue. The perforation occurs at a point of weakness in the bowel wall where vigorous peristaltic movement produces a large pressure gradient, precipitating rupture of the disease site. When subcutaneous emphysema occurs, it is usually confined to the anterior abdominal wall. From there it passes to the lower extremity via the femoral canal or along the iliopsoas muscle to its insertion into the lesser trochanter of the femur. The gas then spreads freely along the fascial planes towards the knee.

The two main types of gas-forming inflammatory processes $^{[11,13]}$ are:

1. Emphysematous cellulitis; accounts for the vast majority of gas-forming infection in hospital practice.

2. Emphysematous myositis (gas gangrene).

Patients usually complain of severe pain, ${ }^{[2]}$ disproportionate pain, ${ }^{[3,6,14]}$ and sometimes describe the sensation as "heavy". ${ }^{[3,15]}$ Patients are usually 
anxious, ${ }^{[2]}$ restless, apprehensive, and tachycardic but normotensive. Gas in the tissue is a late sign; ${ }^{[2,6,15]}$ it may be absent altogether. ${ }^{[2,5,14]}$ Gas in the tissue is neither a sensitive nor a specific sign of clostridial infection. ${ }^{[5]}$ It can be found with E. coli, Streptococcus, Proteus, Enterococcus, Staphylococcus, Klebsiella, Pseudomonas, and Bacteroides species, particularly in patients with diabetes. The skin shows bronze coloration around the area involved, ${ }^{[2,15]}$ and bullae develop that are filled with mousy-smelling fluid containing grampositive rods. Patients usually experience profound metabolic acidosis, and hemolysis caused by the exotoxins may cause fever, hypotension, disseminated intravascular coagulation, and renal failure.

\section{Diagnostic feature}

Gram stain from the bullae renders gram-positive bacilli[i] ${ }^{[6]}$ without spores and very low leukocyte count. The $\operatorname{skin}^{[8]}$ around the bullae is purple, reflecting the vascular compromise that results from diffusion of bacterial toxins in the surrounding tissues. X-ray of the affected limb reveals soft-tissue gas ${ }^{[2,3]}$ [Figure 1]; however, CT scan has been shown to be a more sensitive test. ${ }^{[13]}$ Clinically, the muscle looks dark and cooked, and it does not contract when incised. ${ }^{[5]}$ Other lab results may reveal evidence of hemolysis, hyperbilirubinuria, hyperkalemia, and anemia as a result of the release of toxins. ${ }^{[3]}$

\section{Management}

Initial treatment involves high oxygen concentration and aggressive volume expansion with intravenous isotonic crystalloid fluid. ${ }^{[3]}$ Volume status should be monitored via urinary output and central venous pressure. Blood should be given sparingly, since it will be hemolyzed rapidly. Brummelkamp ${ }^{[16,17]}$ advised delay in transfusion till exotoxin production and hemolysis are brought under control with hyperbaric oxygenation. Vasopressins should be avoided, and severe acidosis should be treated with bicarbonate. Antibiotics should be started, with penicillin $\mathrm{G}$ being the antibiotic of choice, ${ }^{[4,5,7,13,15]}$ given in high doses of 20 40 million units. Alternatively, in the event of penicillin allergy, cephalothin, clindamycin, or metronidazole can be used. Sodium penicillin is preferable to potassium penicillin ${ }^{[2,3,13]}$ because the patient is already at risk of hyperkalemia from tissue breakdown.

The use of hyperbaric oxygen for the treatment of gas gangrene remains controversial. ${ }^{[7]}$ Brummelkamp reported that $21(81 \%)$ of 26 patients with clostridial infection who received hyperbaric oxygen survived. ${ }^{[8,16]}$ Results from more recent study showed survival of $70 \%$ of patients treated with hyperbaric oxygen and $30 \%$ not treated with hyperbaric oxygen. The rationale behind this treatment is that, due to the hypovascularity of the infected site, an extremely high concentration of dissolved oxygen is necessary to raise the tissue $\mathrm{pO}_{2}$. Hyperbaric oxygen is believed to reduce the general toxicity of circulating clostridial toxins ${ }^{[8]}$ and to limit the spread of infection. ${ }^{[2]}$ In addition, hyperbaric oxygen reduces the spore granulation rate and aids eradication of the organism both in vitro and in vivo. ${ }^{[15]}$ The a toxin production is suppressed at an oxygen tension of $250 \mathrm{mmHg}$. This is achieved by the production of oxygen free radicals..$^{[8]}$ Hyperbaric oxygen is also believed to protect the viability of healthy tissue surrounding an area of progressive necrosis. The accepted treatment now is five hyperbaric sessions at three atmospheric pressure within the first $48 \mathrm{~h},{ }^{[3]}$ up to a total of seven to ten sessions.

\section{Surgical treatment}

Surgery remains the critical life-saving intervention and should not be delayed in the interest of transferring a patient to a facility with hyperbaric oxygen. ${ }^{[7]}$ In the absence of adequately debrided wound, antibiotic will not prevent gas gangrene. ${ }^{[15]}$ Surgery ranges from simple fasciotomy to radical debridement/ amputation. ${ }^{[3,4]}$ In a study on dogs, Domello ${ }^{[18]}$ showed that surgery alone or with hyperbaric oxygen left no survivors, whereas surgery with antibiotics left $70 \%$ survivors. Antibiotics alone left $50 \%$ survivors.

There is a better outcome when patients undergo one hyperbaric session prior to initial debridement, and further debridement can be planned between subsequent hyperbaric treatments. The deferment has the following advantages:

1. The patient has better improved general condition.

2. Surgery is limited to the removal of necrotic tissue alone.

3. Necrotic tissue is better demarcated. ${ }^{[2]}$

When fasciotomy is necessary, the procedure should always be performed prior to hyperbaric $\mathrm{O}_{2}$ treatment. ${ }^{[7]}$ Even if the diagnosis is in doubt, it is better to begin antibiotic and hyperbaric oxygen treatment promptly rather than to take a wait-and-see approach ${ }^{\left[{ }^{[15]} \text { Antitoxin }\right.}$ has been shown to be of no value in preventing the spread of clostridial infection.

Finally, patients who do survive should be screened for colonic or hematologic malignancy. ${ }^{[4]}$

In conclusion, clostridial myonecrosis is a rapidly spreading infection which is fatal unless recognized early. Purple discoloration in a limb of a sick patient, with or without crepitation, should be taken as a sinister sign, and early aggressive treatment with 
fluids, oxygen, antibiotics, and surgical debridement/ amputation should be instituted as soon as the diagnosis is suspected.

\section{Financial support and sponsorship}

Nil.

\section{Conflicts of interest}

There are no conflicts of interest.

Patient consent

Patient consent was obtained from the patient.

\section{Ethics approval}

Ethics approval was obtained prior to the commencement of the study.

\section{REFERENCES}

1. Cline KA, Turnbull TL. Clostridial myonecrosis. Ann Emerg Med 1985;14:459-66.

2. Kizer KW, Ogle LC. Occult clostridial myonecrosis. Ann Emerg Med 1981;10:307-11

3. Corey EC. Nontraumatic gas gangrene: case report and review of emergency therapeutics. J Emerg Med 1991;9:431-6.

4. Hawkins C, Riley JL. Spontaneous gas gangrene: an unusual complication of colonic carcinoma. Clin Oncol ( $R$ Coll Radiol) 1997:9:184-5.

5. Kornbluth AA, Danzig JB, Bernstein LH. Clostridium septicum infection and associated malignancy. Report of 2 cases and review of the literature. Medicine (Baltimore) 1989;68:30-7.

6. Hallock GG. Delayed ante mortem diagnosis of adenocarcinoma of the caecum presenting as lower extremity gas gangrene. Dis Colon Rectum 1984;27:131-3.

7. Valentine EG. Nontraumatic gas gangrene. Ann Emerg Med 1997;30:109-11.

8. Stevens DL, Musher DM, Watson DA, Eddy H, Hamill RJ, Gyorkey F, Rosen H, Mader J. Spontaneous, nontraumatic gangrene due to clostridium septicum. Rev Infect Dis 1990;12:286-96.

9. Jendrzejewski JW, Jones SR, Newcombe RL, Gilbert DN Nontraumatic clostridial myonecrosis. Am J Med 1978;65:542-6.

10. Hallock GG, Morain WD. Metastatic nontraumatic gas gangrene. Contemp Surg 1983;22:38-41.

11. Jager GJ, Rijssen HV, Lamers JH. Subcutaneoeous emphysema of the lower extremity of abdominal origin. Gastrointest Radiol 1990;15:253-8.

12. Nicell P, Tabrisky J, Lindstrom R, Peter M. Thigh emphysema and hip pain secondary to gastrointestinal perforation. Surgery 1975;78:555-9

13. Levi N. An unnecessary femoral amputation? J R Coll Surg Edinb 1998;43:196-7.

14. Salanitri GC, Tauro PG. Clostridium septicum septicaemia with myonecrosis. Australas Radiol 1999;43:256-9.

15. Gibson A, Davis FM. Hyperbaric oxygen therapy in the management of Clostridium perfringens infections. N Z Med J 1986:99:617-20

16. Brummelkamp WH, Boerema I, Hoogendy L. Treatment of clostridial infection with hyperbaric oxygen drenching: a report on 26 cases. Lancet 1963;1:235-8.

17. Brummelkamp WH. Treatment of injections with clostridium welchi by oxygen therapy at 3 atmospheres: a report on 37 cases, in Boerama. Amsterdam: Elsevier Publishing Company, 1964.

18. Domello FJ, Haglin JJ, Hichcock CR. Comparative study of experimental Clostridium perfringens infection in dogs treated with antibiotics, surgery and hyperbaric oxygen. Surgery 1973;73:936-41. 